\title{
Pristimerin overcomes adriamycin resistance in breast cancer cells through suppressing Akt signaling
}

\author{
GUI'E XIE $^{1 *}$, XINPEI YU ${ }^{2,3 *}$, HUICHAO LIANG ${ }^{4}$, JINGSONG CHEN $^{4}$, \\ XUEWEI TANG ${ }^{4}$, SHAOQING WU ${ }^{4}$ and CAN LIAO ${ }^{4}$ \\ ${ }^{1}$ KingMed School of Laboratory Medicine, Guangzhou Medical University, Guangzhou, Guangdong 510182; \\ ${ }^{2}$ Cancer Center; ${ }^{3}$ Traditional Chinese Medicine-Integrated Hospital, Southern Medical University, \\ Guangzhou, Guangdong 510315; ${ }^{4}$ Guangzhou Women and Children's Medical Center, \\ Guangzhou Medical University, Guangzhou, Guangdong 510623, P.R. China
}

Received January 3, 2015; Accepted February 5, 2016

DOI: $10.3892 / \mathrm{ol} .2016 .4335$

\begin{abstract}
Breast cancer remains a major public health problem worldwide. Chemotherapy serves an important role in the treatment of breast cancer. However, resistance to chemotherapeutic agents, in particular, multi-drug resistance (MDR), is a major cause of treatment failure in cancer. Agents that can either enhance the effects of chemotherapeutics or overcome chemoresistance are urgently needed for the treatment of breast cancer. Pristimerin, a quinonemethide triterpenoid compound isolated from Celastraceae and Hippocrateaceae, has been shown to possess antitumor, anti-inflammatory, antioxidant and insecticidal properties. The aim of the present study was to investigate whether pristimerin can override chemoresistance in MCF-7/adriamycin (ADR)-resistant human breast cancer cells. The results demonstrated that pristimerin indeed displayed potent cytocidal effect on multidrug-resistant MCF-7/ADR breast cancer cells, and that these effects occurred through the suppression of Akt signaling, which in turn led to the downregulation of antiapoptotic effectors and increased apoptosis. These findings indicate that use of pristimerin may represent a potentially promising approach for the treatment of ADR-resistant breast cancer.
\end{abstract}

Correspondence to: Dr Gui'e Xie, KingMed School of Laboratory Medicine, Guangzhou Medical University, 195 Dongfeng West Road, Guangzhou, Guangdong 510182, P.R. China

E-mail: guier2003@126.com

Dr Shaoqing Wu, Guangzhou Women and Children's Medical Center, Guangzhou Medical University, Guangzhou, Guangdong 510623, P.R. China

E-mail: wushaoqing2014@126.com

*Contributed equally

Key words: pristimerin, breast cancer, chemoresistance, Akt

\section{Introduction}

Breast cancer is the most frequently diagnosed cancer and the leading cause of cancer-associated mortality among females $(1,2)$, accounting for $23 \%$ of the total cancer cases and $14 \%$ of cancer mortalities (3). Chemotherapy serves an important role in the treatment of breast cancer. However, resistance to chemotherapeutic agents, in particular, multi-drug resistance (MDR), is an major cause of treatment failure in cancer. The MDR mechanisms are complicated, involving increased drug efflux, reduced drug uptake, altered metabolism of drugs, altered expression of drug targets, reduced affinity of drug targets, activation of the detoxification system, enhanced repair of drug-induced defects and blocked apoptosis (4). Hence, identification of novel and effective agents that reverse drug resistance in breast cancer is urgently needed.

Pristimerin is a quinonemethide triterpenoid compound isolated from Celastraceae and Hippocrateaceae and has long been used as anti-inflammatory, antioxidant, antimalarial and insecticidal agents (5). In addition, it has been reported that pristimerin has promising clinical potential as both a therapeutic and chemopreventive agent for various types of cancer such as pancreatic cancer, glioma, leukemia, cervical cancer, prostate cancer and breast cancer (6-11). Pristimerin has been shown to induce cell death via a number of several mechanisms, including proteosome inhibition, caspase activation, inhibition of cell cycle progression, and suppression of antiapoptotic nuclear factor kappa $\mathrm{B}(\mathrm{NF}-\kappa \mathrm{B})$ and Akt signaling pathways $(6,9)$. In breast cancer, it has been reported that pristimeirin induces apoptotic cell death in MDA-MB-231 breast cancer cells in a caspase-dependent manner (11). In addition, prestimerin has been demonstrated to inhibit the migration and invasion of breast cancer cells (12). However, to the best of our knowledge, the function of pristimerin on chemoresistance in human breast cancer has not yet been investigated.

The present study aims to address whether pristimerin can reverse chemoresistance in human breast cancer cells. The results demonstrated that pristimerin indeed suppressed the proliferation of ADR-resistant MCF-7/ADR breast cancer cells, and that these effects occurred through the suppression of Akt signaling, which in turn led to the downregulation 
of antiapoptotic effectors and increased apoptosis. These findings indicate that pristimerin may have potential in the treatment of ADR-resistant breast cancer.

\section{Materials and methods}

Chemicals. Pristimerin and ADR were obtained from Sigma-Aldrich (St. Louis, MO, USA) and dissolved in dimethyl sulfoxide (DMSO) to give a stock solution of $1.0 \mathrm{mM}$ and stored at $-20^{\circ} \mathrm{C}$ in small aliquots.

Cell culture. MCF-7 and MCF-7/ADR human breast cancer cells were purchased from Nanjing KeyGen Biotech. Co. Ltd. (Nanjing, China). The cells were grown in Dulbecco's modified Eagle medium (Invitrogen ${ }^{\mathrm{TM}}$; Thermo Fisher Scientific, Inc., Waltham, MA, USA) supplemented with $10 \%$ fetal bovine serum (HyClone; GE Healthcare Life Sciences, Logan, UT, USA). MCF-7/ADR cells were cultured in the above medium containing $1.0 \mu \mathrm{M}$ ADR in order to maintain the ADR-resistant phenotype, upon treatment with or without pristimerin.

Cell viability analysis. Cells were seeded in 96-well flat-bottom plates at a density of $1 \times 10^{4}$ cells per well and cultured in a humidified incubator for $24 \mathrm{~h}$ in the absence or presence of the indicated concentrations of pristimerin and $1.0 \mu \mathrm{M}$ ADR for additional $48 \mathrm{~h}$. Cell viability was measured by using the MTS assay as described previously (13). The concentration of drug that inhibited cell survival by $50 \%\left(\mathrm{IC}_{50}\right)$ was calculated by Bliss's software (14). The resistance factor (RF) was determined from the ratio of the $\mathrm{IC}_{50}$ for MCF-7/ADR to the $\mathrm{IC}_{50}$ for the sensitive cell line MCF-7. The data are presented as mean \pm standard deviation from 3 independent experiments.

Annexin V-FITC (fluorescein isothiocyanate)/propidium iodide (PI) staining assay. MCF-7/ADR cells ( $2 \times 10^{5}$ cells/well) were seeded in $60 \mathrm{~mm}$ plates and cultured in a humidified incubator for $24 \mathrm{~h}$ before treatment with the indicated concentrations $(0,0.5$ or $1.0 \mu \mathrm{M})$ of pristimerin and $1.0 \mu \mathrm{M}$ ADR for an additional $8 \mathrm{~h}$. Apoptosis was determined by flow cytometry using Annexin V-FITC Apoptosis Detection Kit II (BD Pharmingen, San Diego, CA, USA), as described previously (13), and a BD FACSCanto ${ }^{\mathrm{TM}}$ II (BD Biosciences, San Jose, CA, USA).

Terminal deoxynucleotidyl transferase-mediated dUTP nick end labeling (TUNEL) assay. MCF-7/ADR cells were plated at a density of $1 \times 10^{5}$ cells per well in 24-well flat-bottom plates, treated with $1.0 \mu \mathrm{M}$ pristimerin and $1.0 \mu \mathrm{M}$ ADR for $24 \mathrm{~h}$. The TUNEL assay for in situ detection of apoptosis was performed by using the DeadEnd ${ }^{\mathrm{TM}}$ Fluorometric TUNEL System assay kit (Promega Corporation, Madison, WI, USA) as previously reported (13).

Caspase activity assay. After treatment of pristimerin with the indicated concentrations $(0,0.5$ or $1.0 \mu \mathrm{M})$ and $1.0 \mu \mathrm{M}$ ADR for $24 \mathrm{~h}$, activity of caspase- 8 and -9 was measured using a Caspase Colorimetric Assay kit (Nanjing KeyGen Biotech. Co. Ltd.) according to the manufacturer's protocol as described previously (13).
Table I. $\mathrm{IC}_{50}$ values of pristimerin on MCF-7/ADR cells.

\begin{tabular}{lccrrr}
\hline \multirow{2}{*}{ Cell lines } & \multicolumn{2}{c}{$\mathrm{ADR}$} & & \multicolumn{2}{c}{ Pristimerin } \\
\cline { 2 - 3 } & $\mathrm{IC}_{50}$ & $\mathrm{RF}$ & & $\mathrm{IC}_{50}$ & $\mathrm{RF}$ \\
\hline MCF-7 & 1.12 & - & & 0.59 & - \\
MCF-7/ADR & 16.29 & 14.54 & & 0.43 & 0.73
\end{tabular}

$\mathrm{IC}_{50}$, the concentration of drug that inhibited cell survival by $50 \%$; ADR, adriamycin; RF, resistance factor.

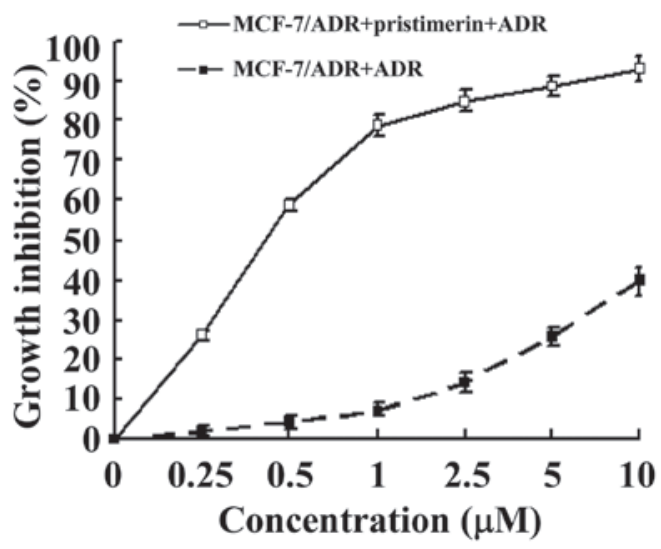

Figure 1. Effect of pristimerin on the growth of MCF-7/ADR cells. Cells were seeded in 96-well plates and incubated with the indicated concentrations of pristimerin for $48 \mathrm{~h}$ at $37^{\circ} \mathrm{C}$. Cell viabilities were determined by MTS assay. Data points are presented as mean \pm standard deviation of triplicate experiments. ADR, adriamycin.

Western blotting analysis. Following treatment with pristimerin and/or $1.0 \mu \mathrm{M}$ ADR at various concentrations $(0,0.5$ and $1.0 \mu \mathrm{M}$ ) for $48 \mathrm{~h}$, cells in each dish, including dead cells floating in medium, were harvested and lysed in $1 \mathrm{X}$ sampling buffer (Nanjing KeyGen Biotech. Co. Ltd.). Protein concentrations of the lysates were determined using the Pierce Bicinchoninic Acid Protein Assay kit (Thermo Fisher Scientific, Inc.). An aliquot of the denatured supernatant containing $30 \mu \mathrm{g}$ of protein was subjected to $12 \%$ sodium dodecyl sulphate polyacrylamide gel electrophoresis, and subsequently transferred to polyvinylidene fluoride membranes (Millipore, Billerica, MA, USA). After blocking with blocking buffer (Tris-buffered saline containing $5 \%$ non-fat milk) for $1 \mathrm{~h}$ at room temperature, the membranes were incubated overnight at $4^{\circ} \mathrm{C}$ with the following specific primary antibodies: Monoclonal mouse anti-human caspase- 8 (catalog no. 9746), polyclonal rabbit anti-human caspase-9 (catalog no. 9502), polyclonal rabbit anti-human PARP (catalog no. 9542), polyclonal rabbit anti-human p-Akt (Ser473; catalog no. 9271), polyclonal rabbit anti-human Akt (catalog no. 9272), monoclonal rabbit anti-human p-Bad (Ser136; catalog no. 4366), polyclonal rabbit anti-human Bad (catalog no. 9292), polyclonal rabbit anti-human Bcl-xL (catalog no. 2762) and monoclonal mouse anti-human GAPDH (catalog no. 60004-1; dilution, 1:10,000; ProteinTech Group, Inc., Chicago, IL, USA). All the antibodies were purchased from Cell Signaling Technology, Inc. (Danvers, MA, USA) and were diluted at 1:1,000, unless otherwise specified. Further incubation with 

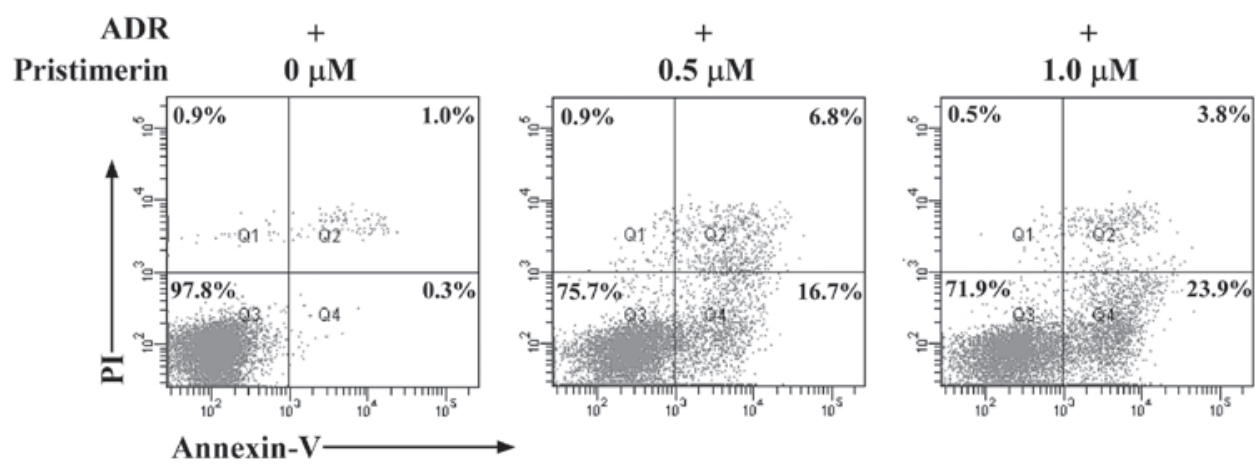

Figure 2. AnnexinV-FITC/PI staining of MCF-7/ADR cells treated with pristimerin. Cells were exposed to different concentrations $(0,0.5,1.0 \mu \mathrm{M})$ of pristimerin and $1.0 \mu \mathrm{M}$ ADR for $8 \mathrm{~h}$. Cells were collected and subjected to Annexin V-FITC/PI staining and analyzed by flow cytometry. PI, propidium iodide; ADR, adriamycin.
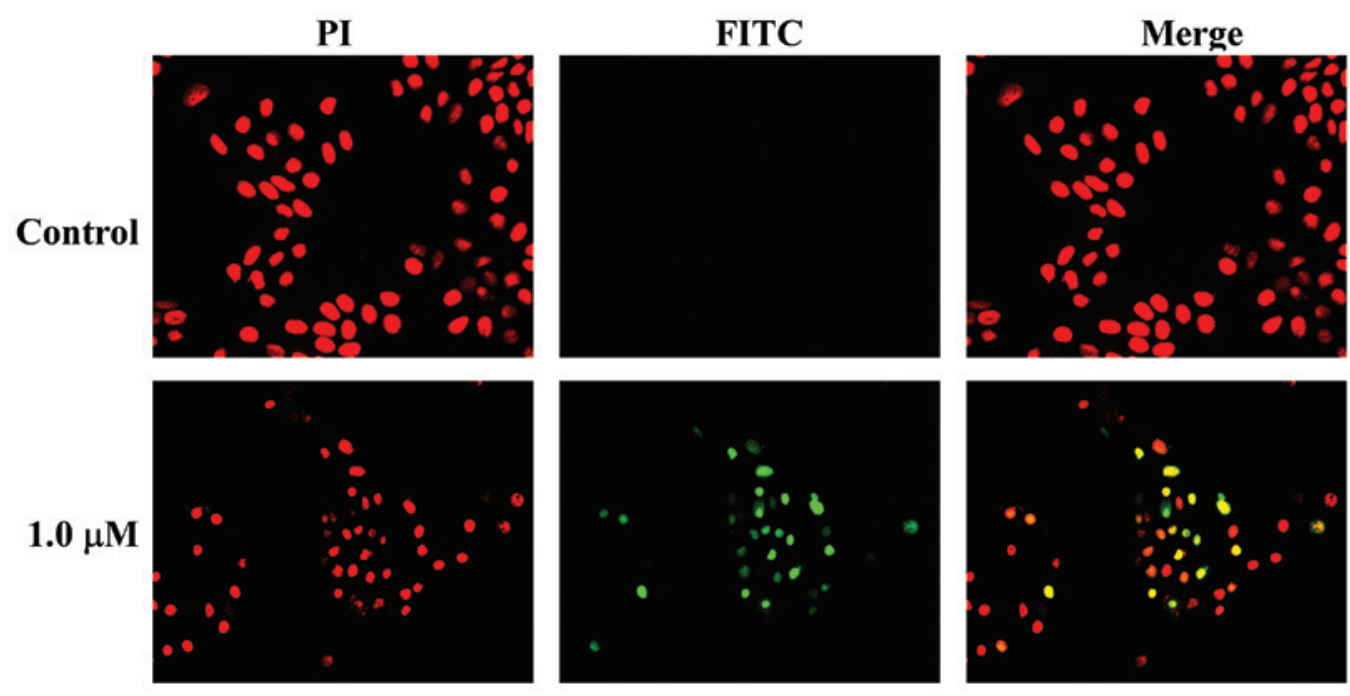

Figure 3. Terminal deoxynucleotidyl transferase-mediated dUTP nick end labeling assay of MCF-7/ADR cells treated with pristimerin. Cells were treated with $1.0 \mu \mathrm{M}$ of pristimerin for $24 \mathrm{~h}$ and labeled with fluorescein-12-dUTP (green) and PI counterstaining (red). ADR, adriamycin.

appropriate horseradish peroxidase-conjugated goat anti-rabbit (catalog. no. SA00001-2; 1:4,000) or goat anti-mouse (catalog no. SA00001-1: 1:4,000) IgG secondary antibodies (ProteinTech Group, Inc.), depending on the primary antibody used, was performed for $1 \mathrm{~h}$ at room temperature. Detection of staining signals was performed using the Pierce Enhanced Chemiluminescence Western Blotting Substrate (Thermo Fisher Scientific, Inc.).

Statistical analysis. The data given in the text are expressed as the mean \pm standard deviation (SD). Comparisons between groups for statistical significance were carried out with a two-tailed Student's $t$-test using SPSS version 17.0 software (SPSS, Inc., Chicago, IL, USA). In all cases, $\mathrm{P}<0.05$ was considered to indicate a statistically significant difference.

\section{Results}

Growth inhibition of ADR-resistant MCF-7 cell by pristimerin. The effect of pristimerin on the growth of the ADR-resistant human breast cancer cell line MCF-7/ADR and the parental sensitive MCF-7 cells was investigated using the MTS assay. After treatment with pristimerin for $48 \mathrm{~h}, \mathrm{MCF}-7 / \mathrm{ADR}$ cells displayed markedly inhibited growth, compared with control cells treated with vehicle, in a dose-dependent manner (Fig. 1). The calculated $\mathrm{IC}_{50}$ values, i.e. the concentrations of pristimerin that inhibited cell survival by $50 \%$, were $0.43 \mu \mathrm{M}$ for MCF-7/ADR and 0.59 $\mu \mathrm{M}$ for MCF-7, respectively. Pristimerin reduced the RF from 14.54 to 0.73 (Table I), which revealed that pristimerin reversed the ADR resistance of MCF-7/ADR cells.

Pristimerin induced apoptosis in MCF-7/ADR cells. To investigate whether pristimerin was able to induce apoptosis in MCF-7/ADR cells, an Annexin V-FITC/PI binding assay was performed. As shown in Fig. 2, after $8 \mathrm{~h}$ treatment with pristimerin at $0.5,1.0 \mu \mathrm{M}$, the percentage of apoptotic MCF-7/ADR cells (Annexin $\mathrm{V}^{+} / \mathrm{PI}^{-}$), markedly increased in a dose-dependent manner. In addition, when the TUNEL assay was used to evaluate apoptosis-induced nuclear DNA fragmentation as a late event during the apoptotic process, a higher amount of TUNEL-positive cells was demonstrated in MCF-7/ADR cells treated for $24 \mathrm{~h}$ with pristimerin at $1.0 \mu \mathrm{M}$ compared to the control (Fig. 3). These results strongly indicated a proapoptotic effect of pristimerin on MCF-7/ADR cells. 
A

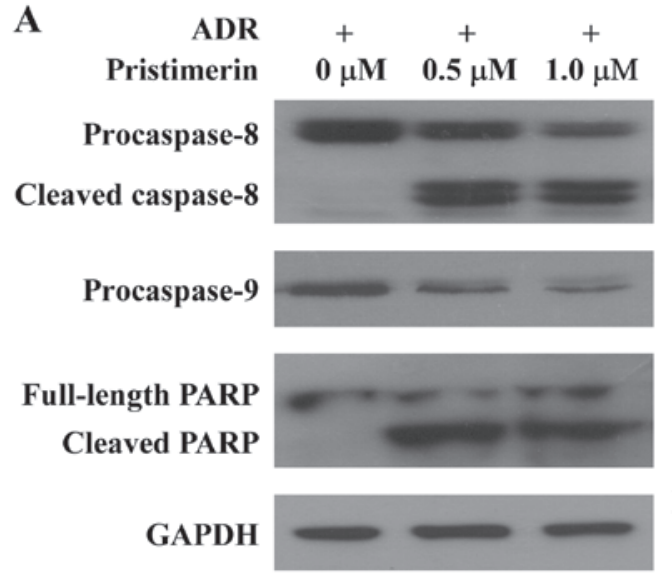

B

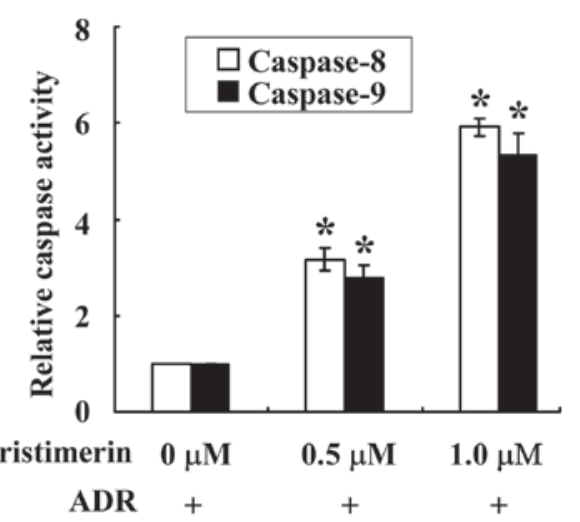

Figure 4. Activation of caspases and PARP in MCF-7/ADR cells by pristimerin. (A) Western blotting analysis of caspases and PARP in MCF-7/ADR cells after pristimerin treatment at different concentrations for $48 \mathrm{~h}$ using antibodies against caspase-8, -9, and PARP. GAPDH was used as an internal control. (B) Enzymatic activities of caspases in pristimerin treated MCF-7/ADR cells, as assessed by colorimetric assay. The fold-increases in the activities of caspases- 8 and -9 were determined by comparison with those of the vehicle-treated control cells. Results are presented as mean \pm standard deviation. ${ }^{*} \mathrm{P}<0.05$; significant differences compared with the control. ADR, adriamycin.

A

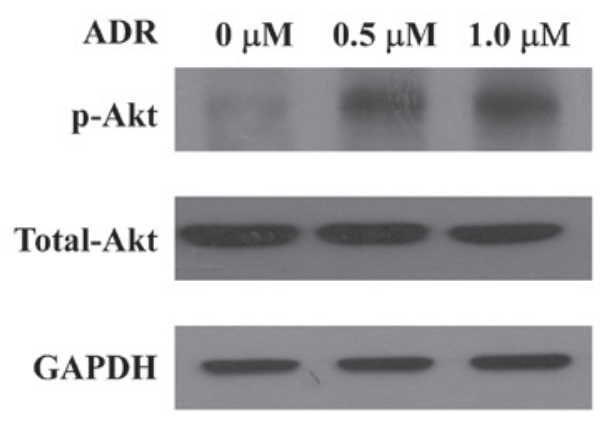

B

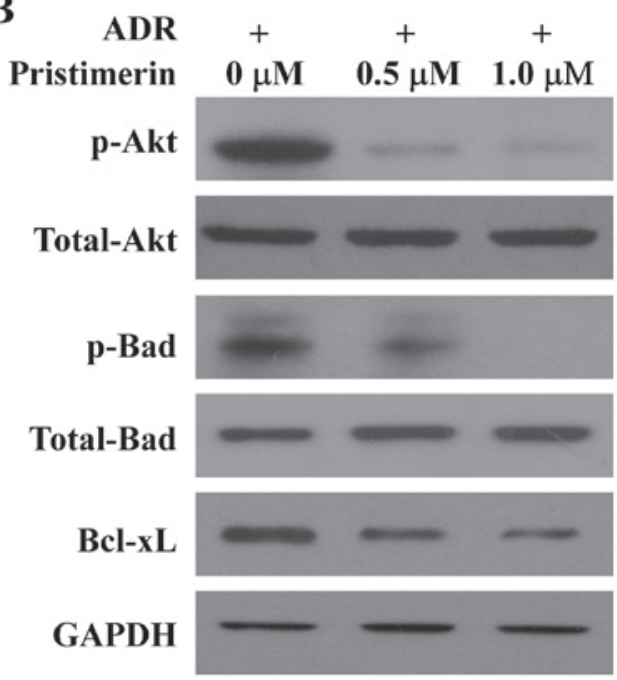

Figure 5. Effect of pristimerin on the Akt signaling pathway in MCF-7/ADR cells. Cells were treated with the indicated concentrations of (A) ADR and (B) pristimerin for $48 \mathrm{~h}$. Western blotting analysis was performed using antibodies against phospho-Akt (Ser473), total Akt, phospho-Bad (Ser 136), total Bad, and Bcl-xL, with GAPDH used as a loading control. ADR, adriamycin; p, phospho.

Activation of both the intrinsic and extrinsic apoptotic pathways by pristimerin in MCF-7/ADR cells. Caspase activation serves a central role in the execution of apoptosis (15). To further understand the apoptotic process in MCF-7/ADR cells, caspase- 9 and -8 , the proapoptotic caspases in intrinsic and extrinsic apoptotic pathways, respectively, were examined using western blotting and enzymatic activity assays. As shown in Fig. 4A, pristimerin treatment dose-dependently activated the cleavage of inactive procaspase- 9 and -8 in MCF-7/ADR cells. Consistent with the results of western blotting analysis, the enzymatic activity of caspase- 9 and -8 markedly increased in a dose-dependent manner after treatment with pristimerin at $0.5,1.0 \mu \mathrm{M}$ (Fig. 4B). The effector molecule PARP was cleaved into the $85 \mathrm{kDa}$ fragment after pristimerin treatment in MCF-7/ADR cells (Fig. 4A). These results indicated that apoptosis induced by pristimerin in MCF-7/ADR cells may involve both intrinsic and extrinsic pathways.
Suppression of Akt activation and its downstream substrates by pristimerin. In an effort to further understand the signaling cascade that mediates the proapoptotic effect of pristimerin on MCF-7/ADR cells, whether pristimerin modulates the activation of Akt was next investigated. As shown in Fig. 5, Akt was found to be constitutively activated upon treatment with ADR in MCF-7/ADR cells (Fig. 5A), and pristimerin treatment suppressed constitutively phosphorylated Akt levels in a dose-dependent manner without an effect on total Akt expression (Fig. 5B). Bad is a proapoptotic member of the Bcl-2 family. Dephosphorylated Bad forms a heterodimer with Bcl-2 and Bcl-xL. When Bad is phosphorylated by Akt, it promotes binding of Bad to 14-3-3 proteins (16). This leaves Bcl-2 free to inhibit apoptosis and then promote cell survival. Consistent with the suppression of Akt activation, pristimerin treatment reduced phosphorylation of Bad (Ser136) dose-dependently without an effect on total Bad expression (Fig. 5B). In addition, 
Bcl-xL, another antiapoptotic effector downstream of Akt signaling, was found to be downregulated upon treatment with pristimerin in MCF-7/ADR cells (Fig. 5B).

\section{Discussion}

Chemoresistance remains a major therapeutic challenge in breast cancer. In recent years, increasing effort has been focused on the identification of novel anticancer agents. Triterpenoids, possessing a wide range of unique biological activities, have received the most attention in this area (17). Triterpenoids such as 2-cyano-3, 12-dioxooleana-1, 9-dien-28-oic acid (CDDO), its methyl ester (CDDO-Me), betulinic acid and the ginsenosides, have shown promising anticancer activities and are presently being evaluated in clinical trials for the treatment of leukemia, lymphoma, and solid tumors (18). Pristimerin is a triterpenoid isolated from Celastraceae and Hippocrateaceae and has been previously demonstrated to be a promising therapeutic agent against several types of cancer. Previous studies have shown that pristimerin is able to inhibit breast cancer cell proliferation and migration $(11,12)$. However, the function of pristimerin on MDR in human breast cancer has yet to be elucidated. Therefore, in the present study, the effect of pristimerin on MCF-7/ADR human breast cancer MDR cells was investigated.

Consistent with previous reports, in the present study, pristimerin displayed substantial cytotoxic activity on MCF-7 breast cancer cells, with greater potency than that of other triterpenoids for solid organ malignancies such as CDDO or betulinic acid $(8,19)$, which are currently in clinical trials. For MDR MCF-7/ADR cells $\left(\mathrm{IC}_{50}, 0.43 \mu \mathrm{M}\right)$, pristimerin displayed equally, if not more, effective cytocidal effects as compared with that against sensitive MCF-7 cells $\left(\mathrm{IC}_{50}, 0.59 \mu \mathrm{M}\right)$, which indicates that pristimerin is able to reverse the ADR resistance of MCF-7/ADR cells.

The precise molecular mechanism underlying chemoresistance in breast cancer is not clear, but the activation of the phosphoinositide-3-kinases (PI3K)/Akt signaling and dysregulation of apoptosis have been implicated as the major players involved in chemoresistance (19-22). Apoptosis is a key mechanism by which anticancer drugs kill tumor cells, which is a cellular suicide program through the mitochondrial (intrinsic) or death receptor pathways (extrinsic). In the extrinsic pathway, binding of death ligands (e.g. TNF- $\alpha$, FasL, TRAIL) with their cognate receptors activates initiator caspase-8 which then cleaves and activates effector caspases-3, -6 , and -7 leading to apoptosis. In mitochondrial or intrinsic pathway, undefined signals induce release of cytochrome $\mathrm{c}$ from mitochondria, which in conjunction with Apaf-1 causes activation of initiator caspase-9. Activated caspase-9, in turn, activates effector caspases-3, -6, and -7. In the present study, it was confirmed that pristimerin induced significant apoptosis in MCF-7/ADR cells through both the extrinsic and intrinsic apoptotic pathways as evidenced by activation of caspases, PARP cleavage, Annexin V-positive binding and TUNEL-positive staining.

Chemoresistance in several types of cancer has been linked to activation of the PI3K/Akt signaling pathway. The $\mathrm{PI} 3 \mathrm{~K} / \mathrm{Akt}$ pathway is involved in the regulation of a number of cellular processes including cell death and survival, cell proliferation, protein synthesis and cell metabolism $(23,24)$. Accumulating evidence has indicated that activation of the PI3K/Akt pathway may confer acquired resistance to chemotherapeutic drugs with different mechanisms of actions: ADR, mitoxantrone, 5-fluorourocil, etoposide, camptothecin, etc $(20,21)$. In breast cancer cells, activation of Akt by HER2/PI3K is involved in conferring broad-spectrum chemoresistance. Therefore, Akt is considered to be a novel target to develop therapeutic strategy to improve the outcome of breast cancer chemotherapy (25). In agreement with previous reports, the present study revealed that Akt was indeed constitutively activated upon ADR treatment in MCF-7/ADR cells. The ability of pristimerin to suppress the Akt signaling pathways has been previously reported in a number of sensitive cancer cell lines $(6,26)$. For ADR-resistant MCF-7/ADR cells, it was demonstrated that pristimerin treatment also resulted in a dose-dependent reduction in phospho-Ser473-Akt without an effect on total Akt expression. Bad and Bcl-xL are two crucial mediators downstream of Akt signaling, and dysregulation of Bad and Bcl-xL has been linked to chemoresistance (27-30). In the present study, in parallel with the observed reduction of Akt phosphorylation, phosphorylation of Bad was inhibited and Bcl-xL was downregulated in response to pristimerin treatment in MCF-7/ADR cells.

In conclusion, the results of the present study indicate that pristimerin is a potent apoptosis inducer in breast cancer cells resistant to ADR treatments, and its mechanism is relevant with the suppression of Akt signaling, which indicates the therapeutic value of pristimerin as a potential MDR reversing agent for breast cancer chemotherapy.

\section{Acknowledgements}

The present study was supported by a grant from the National Natural Science Foundation of China (grant no. 81101682).

\section{References}

1. Friedenreich CM: Physical activity and breast cancer: Review of the epidemiologic evidence and biologic mechanisms. Recent Results Cancer Res 188: 125-139, 2011.

2. Zheng L, Zhou B, Meng X, Zhu W, Zuo A, Wang X, Jiang R and Yu S: A model of spontaneous mouse mammary tumor for human estrogen receptor- and progesterone receptor-negative breast cancer. Int J Oncol 45: 2241-2249, 2014.

3. Jemal A, Bray F, Center MM, Ferlay J, Ward E and Forman D: Global cancer statistics. CA Cancer J Clin 61: 69-90, 2011.

4. Gottesman MM: Mechanisms of cancer drug resistance. Annu Rev Med 53: 615-627, 2002.

5. Dirsch VM, Kiemer AK, Wagner H and Vollmar AM: The triterpenoid quinonemethide pristimerin inhibits induction of inducible nitric oxide synthase in murine macrophages. Eur J Pharmacol 336: 211-217, 1997.

6. Deeb D, Gao X, Liu YB, Pindolia K and Gautam SC: Pristimerin, a quinonemethide triterpenoid, induces apoptosis in pancreatic cancer cells through the inhibition of pro-survival $\mathrm{Akt} / \mathrm{NF}-\kappa \mathrm{B} / \mathrm{mTOR}$ signaling proteins and anti-apoptotic Bcl-2. Int J Oncol 44: 1707-1715, 2014.

7. Yan YY, Bai JP, Xie Y, Yu JZ and Ma CG: The triterpenoid pristimerin induces U87 glioma cell apoptosis through reactive oxygen species-mediated mitochondrial dysfunction. Oncol Lett 5: 242-248, 2013.

8. Tiedemann RE, Schmidt J, Keats JJ, Shi CX, Zhu YX, Palmer SE, Mao X, Schimmer AD and Stewart AK: Identification of a potent natural triterpenoid inhibitor of proteosome chymotrypsin-like activity and NF-kappaB with antimyeloma activity in vitro and in vivo. Blood 113: 4027-4037, 2009. 
9. Byun JY, Kim MJ, Eum DY, Yoon CH, Seo WD, Park KH, Hyun JW, Lee YS, Lee JS, Yoon MY and Lee SJ: Reactive oxygen species-dependent activation of Bax and poly(ADP-ribose) polymerase-1 is required for mitochondrial cell death induced by triterpenoid pristimerin in human cervical cancer cells. Mol Pharmacol 76: 734-744, 2009.

10. Liu YB, Gao X, Deeb D, Brigolin C, Zhang Y, Shaw J, Pindolia K and Gautam SC: Ubiquitin-proteasomal degradation of antiapoptotic survivin facilitates induction of apoptosis in prostate cancer cells by pristimerin. Int J Oncol 45: 1735-1741, 2014.

11. Wu CC, Chan ML, Chen WY, Tsai CY, Chang FR and Wu YC: Pristimerin induces caspase-dependent apoptosis in MDA-MB-231 cells via direct effects on mitochondria. Mol Cancer Ther 4: 1277-1285, 2005.

12. Mu XM, Shi W, Sun LX, Li H, Wang YR, Jiang ZZ and Zhang LY: Pristimerin inhibits breast cancer cell migration by up- regulating regulator of $G$ protein signaling 4 expression. Asian Pac J Cancer Prev 13: 1097-1104, 2012.

13. Xie G, Zhu X, Li Q, Gu M, He Z, Wu J, Li J, Lin Y, Li M, She Z and Yuan J: SZ-685C, a marine anthraquinone, is a potent inducer of apoptosis with anticancer activity by suppression of the Akt/FOXO pathway. Br J Pharmacol 159: 689-697, 2010.

14. Bliss CI: The calculation of the dose-mortality curve. Ann Appl Biol 22: 134-167, 1935.

15. Evan GI and Vousden KH: Proliferation, cell cycle and apoptosis in cancer. Nature 411: 342-348, 2001.

16. Datta SR, Dudek H, Tao X, Masters S, Fu H, Gotoh Y and Greenberg ME: Akt phosphorylation of BAD couples survival signals to the cell-intrinsic death machinery. Cell 91: 231-241, 1997.

17. Setzer WN and Setzer MC: Plant-derived triterpenoids as potential antineoplastic agents. Mini Rev Med Chem 3: 540-556, 2003.

18. Petronelli A, Pannitteri G and Testa U: Triterpenoids as new promising anticancer drugs. Anticancer Drugs 20: 880-892, 2009

19. Clark AS, West K, Streicher S and Dennis PA: Constitutive and inducible Akt activity promotes resistance to chemotherapy, trastuzumab, or tamoxifen in breast cancer cells. Mol Cancer Ther 1: 707-717, 2002.
20. Knuefermann C, Lu Y, Liu B, Jin W, Liang K, Wu L, Schmidt M, Mills GB, Mendelsohn J and Fan Z: HER2/PI-3K/Akt activation leads to a multidrug resistance in human breast adenocarcinoma cells. Oncogene 22: 3205-3212, 2003.

21. Raguz S and Yagüe E: Resistance to chemotherapy: New treatments and novel insights into an old problem. Br J Cancer 99: 387-391, 2008.

22. Kashkar H: X-linked inhibitor of apoptosis: A chemoresistance factor or a hollow promise. Clin Cancer Res 16: 4496-4502, 2010.

23. Katso R, Okkenhaug K, Ahmadi K, White S, Timms J and Waterfield MD: Cellular function of phosphoinositide 3-kinases: Implications for development, homeostasis and cancer. Annu Rev Cell Dev Biol 17: 615-675, 2001.

24. Sui T, Ma L, Bai X, Li Q and Xu X: Resveratrol inhibits the phosphatidylinositide 3-kinase/protein kinase $\mathrm{B} /$ mammalian target of rapamycin signaling pathway in the human chronic myeloid leukemia K562 cell line. Oncol Lett 7: 2093-2098, 2014.

25. Knight ZA and Shokat KM: Chemically targeting the PI3K family. Biochem Soc Trans 35: 245-249, 2007.

26. Gao X, Liu Y, Deeb D, Arbab AS and Gautam SC: Anticancer activity of pristimerin in ovarian carcinoma cells is mediated through the inhibition of prosurvival Akt/NF- $\mathrm{BB} / \mathrm{mTOR}$ signaling. J Exp Ther Oncol 10: 275-283, 2014.

27. Marchion DC, Cottrill HM, Xiong Y, Chen N, Bicaku E, Fulp WJ, Bansal N, Chon HS, Stickles XB, Kamath SG, et al: BAD phosphorylation determines ovarian cancer chemosensitivity and patient survival. Clin Cancer Res 17: 6356-6366, 2011.

28. Sun Q, Yogosawa S, Iizumi Y, Sakai T and Sowa Y: The alkaloid emetine sensitizes ovarian carcinoma cells to cisplatin through downregulation of bcl-xL. Int J Oncol 46: 389-394, 2015.

29. Villedieu M, Louis MH, Dutoit S, Brotin E, Lincet H, Duigou F, Staedel C, Gauduchon P and Poulain L: Absence of Bcl-xL down-regulation in response to cisplatin is associated with chemoresistance in ovarian carcinoma cells. Gynecol Oncol 105: 31-44, 2007.

30. Williams J, Lucas PC, Griffith KA, Choi M, Fogoros S, Hu YY and Liu JR: Expression of Bcl-xL in ovarian carcinoma is associated with chemoresistance and recurrent disease. Gynecol Oncol 96: 287-295, 2005. 